\title{
Design and verification of micro/nano probes for coordinate measuring machines (Withdrawal Notice)
}

Peng Xu, Rui Jun Li, Xiao Yu Feng, Kuang Chao Fan

Peng Xu, Rui Jun Li, Xiao Yu Feng, Kuang Chao Fan, "Design and verification of micro/nano probes for coordinate measuring machines (Withdrawal Notice)," Proc. SPIE 12059, Tenth International Symposium on Precision Mechanical Measurements, 120591Q (19 November 2021); doi: $10.1117 / 12.2613440$

SDIE Event: Tenth International Symposium on Precision Mechanical Measurements, 2021, Qingdao, China 


\section{Design and venific ation of mic ro/nano probes for coordinate measuring mac hines (withdrawal notice)}

Peng Xu, Rui J un Li, Xiao Yu Feng, Kuang Chao Fan

Hefei Univ. of Technology

Proceedings Volume 12059, Tenth Intemational Symposium on Precision Mechanic al Measurements:

120591Q (2021) http://dx.doi.org/10.1117/12.2613440

Event Tenth Intemational Symposium on Precision Mechanic al Measurements, Q ingdao, China

Online Publication Date: 19 November 2021

Withdrawn from Publication: 30 November 2021

Publisher's Note: This paper, orig ina lly published on 19 November 2021 was withd ra wn on 30 November 2021 pera uthor request. 